\title{
ELEMENTOS EMERGENTES EN LA NUEVA CONFIGURACIÓN DE LAS FUNCIONES SUSTANTIVAS DE LA UNIVERSIDAD
}

\author{
Claudio Roberto Perdomo Interiano \\ Centro Regional San Pedro Sula \\ Universidad Pedagógica Nacional Francisco Morazán \\ crperdomo20@hotmail.com
}

\section{RESUMEN}

En este artículo se presentan una serie de reflexiones sobre la resignificación de las funciones sustanciales de la universidad: docencia, investigación y vinculación con la sociedad, tomando en cuenta algunas de las tendencias y características de la sociedad en el siglo XXI; lo cual, lleva a concebir la actividad universitaria como un quehacer multicontextualizado y cambiante. En tal sentido, se plantea que las distintas formas de trabajo que se desarrollan en los escenarios de su competencia, deben establecerse sobre la base de criterios tales como la pluralidad, flexibilidad, integración e innovación permanente.

Asimismo, se sostiene que los enmarcamientos aúlicos y la autodeterminación de los recintos académicos que en el pasado constituyeron fuentes y mecanismos para la difusión de normas de autoridad a través de las cuales se ejercía el monopolio del conocimiento, han entrado en la inevitable decadencia, conformándose como esa nueva banalidad que sucumbe ante la fuerza transformadora de la cognición postmoderna y de la irreverente actividad de la investigación científica.

En las líneas finales del artículo, se presentan tres propuestas para la nueva configuración de las funciones sustanciales de la universidad: el fomento del trabajo interdisciplinario en las distintas dimensiones del desarrollo y transformación de la cultura académica, la bioética como espacio de trabajo transdisciplinario y el fortalecimiento de la relación heurística entre la docencia, la investigación y la vinculación con la sociedad.

\section{PALABRAS CLAVE}

Docencia, investigación, vinculación social, interdisciplinariedad, transdisciplinariedad, integración, heurística, cultura académica, globalización, innovación permanente. 
Recibido 27 de junio de 2016 /Aceptado 1 de noviembre de 2016 ABSTRACT

This article presents a series of reflections about the resignification of the substantial functions of the university: teaching, research, and engagement with society, taking into account some of the tendencies and characteristics of the society on the $21^{\text {st }}$ century, which leads us to conceive the university activity as a multi-contextualized and changing task. In this sense, it is said that the different work forms that are developed in the scenarios of its competence, must be established on the basis of criteria such as plurality, flexibility, integration and permanent innovation.

In addition, it submits that the classroom frameworks and the self determination of the academic enclosures that in the past constituted sources and mechanisms for the diffusion of authority rules, through which they exercised the knowledge monopoly, they have entered into the inevitable decadence, conforming the new banality that succumbs before the transforming power of post-modern cognition and the irreverent activity of scientific research.

On the final lines of the article, three proposals are presented for the new configuration of the university substantial functions: the promotion of interdisciplinary work on the different dimensions of the academic culture development and transformation, the bioethics as transdisciplinary workspace, and the strengthening of the heuristic relationship between teaching, research, and the engagement with society.

\section{KEY WORDS}

Teaching, research, social engagement, transdisciplinarity, integration, heuristics, academic culture, globalization, and permanent innovation.

\section{CONTEXTO}

La vida universitaria de nuestro tiempo, puede ser comprendida desde una visión ontológica que se construye a través de interpretaciones a los cambios acelerados y al aparecimiento inusitado de formas convencionales y no convencionales, o de la 
combinación entre ellas, para llevar a cabo las funciones sustantivas que histórica y culturalmente han constituido su identidad institucional.

De esta manera, al intentar llevar su comprensión al nivel teórico, encontramos que el marco ontológico al que acudimos para construir su fundamentación, permite desplegar la actividad universitaria como un quehacer multicontextualizado y descentrado; por tanto, con incidencias inacabadas que condicionan a la docencia, la investigación y la vinculación con la sociedad, requiriendo que las mismas se funden entre la plasticidad determinada de un mundo en constante transformación y el rigor que la didáctica y la investigación científica deben poseer como condición esencial para desarrollar los procesos de enseñanza y la producción de conocimientos; así como, para analizar y ofrecer soluciones a los problemas presentes en la realidad socio - natural en la cual se halla situada.

En un mundo anclado en las interdependencias y fluctuaciones, las funciones sustantivas de la universidad se presentan como formas de trabajo que deben establecerse sobre la base de criterios tales como la pluralidad, integración, flexibilidad e innovación permanente; en tal sentido, su configuración no sólo debe comprenderse como el simple reflejo de las condiciones de los entornos y de las manifestaciones internacionales en los que aparecen una y otra vez, elementos emergentes que atraen, motivan, angustian, desafían y provocan la necesidad del cambio; sino, como identidades que se instauran a través de la construcción crítica, la indagación e invención en las concepciones y procesos que tienen lugar de manera intrínseca y extrínseca en su actividad permanente.

Como afirma Gimeno Sacristán (2001:76):

La globalización significa el establecimiento de interconexiones entre países o partes del mundo, intercambiándose las formas de vivir de sus gentes, lo que estas piensan o hacen, generándose interdependencias en la economía, la defensa, la política, la cultura, la ciencia, la tecnología, las comunicaciones, etc.

Esto ha llevado a la adopción de un pensamiento autocrítico en la comunidad académica, pues se ha comprendido que en la actual circunstancia histórica, la autosuficiencia carece de sentido y de contexto, fundando en su lugar, relaciones dialógicas para compartir experiencias, tejer redes y establecer relaciones de colaboración que permitan cumplir y anticiparse a las demandas de la sociedad.

Una de las justificaciones que posee esta nueva forma de ver la cuestión universitaria, está en que: 
La ciencia viene sufriendo los cambios de la época y no está exenta de los ritmos sociales ni del uso extendido del conocimiento, lo que se refleja en la idea generalizada de formar una amplia masa de investigadores que incidan en el desarrollo social y en los entornos locales. Como consecuencia, se produce una idea generalizada sobre la importancia de hablar de ciencia en la escuela, en la comunidad y en la interacción social (Rojas, 2011).

Desde esta perspectiva, se han instaurado procesos como el que da lugar al tratado de Bolonia en el año 1999, cuyos objetivos han llegado a tener impacto en las instituciones de educación superior de América Latina y El Caribe. En el caso de la región centroamericana, se puede reconocer que paulatinamente se ha venido transitado desde universidades cuyo énfasis ha estado en la enseñanza, difusión del conocimiento y el trabajo parcelado de facultades y departamentos a formas de trabajo basadas en la integración, que aunque todavía siguen siendo incipientes, marcan pasos importantes para la recomposición teórica y práctica de la docencia, la investigación y la vinculación con la sociedad.

\section{CONCEPTUALIZACIÓN}

Las distintas perspectivas mediante las que se construyen los significados que ostenta la educación superior, permiten afirmar además, que se trata de un escenario esencialmente dinámico, en el que si bien, predomina la idea acerca de su papel fundamental en la producción, distribución, aprendizaje y aplicación del conocimiento; también se hace presente la necesidad de contextualizar el cumplimiento de sus funciones sustantivas en la vida comunitaria y en la promoción de relaciones responsables con el medio ambiente.

Como lo expresa Villagra:

Más allá de la centralidad propia de la acción transmisiva que tiene lugar en las aulas, las prácticas de enseñanza, al estar encuadradas dentro de los marcos políticosacadémicos reguladores del nivel superior universitario, lejos de circunscribirse a la visión tradicional de una docencia encapsulada en el aula, deben resignificarse en torno a la aspirada articulación con las funciones de investigación y extensión, lo que les confiere una cierta particularidad como objeto de análisis didáctico (2011:31).

La integración de la docencia, investigación y vinculación con la sociedad, debe fundarse en elementos transversales que posibiliten las transiciones e intercambios continuos de los marcos conceptuales, metodologías, actividades y hallazgos propios de cada una de ellas, con la finalidad de lograr su reestructuración en los tiempos y espacios 
socio - universitarios; esto requiere, el paso de las estrategias analíticas a la aplicación de otro tipo de estrategias más abiertas e interdependientes en el desarrollo del trabajo científico, pedagógico y comunitario; procurando que los modos de actuación e interpretación de los actores y comunidades académicas coexistan entre la identidad y la diferencia de los saberes y propiedades particulares de la realidad.

Los enmarcamientos aúlicos y la autodeterminación de los recintos académicos que en el pasado constituyeron fuentes y mecanismos para la difusión de normas de autoridad a través de las cuales se ejercía el monopolio del conocimiento, o que en otros casos, se presentaron como formas de intimidación a la curiosidad y a la pregunta, han entrado en la inevitable decadencia, conformándose como esa nueva banalidad que sucumbe ante la fuerza transformadora de la cognición postmoderna y de la irreverente actividad de la investigación científica.

En la Conferencia Mundial sobre Educación Superior celebrada con el nombre de la nueva dinámica de la educación superior y la investigación para el cambio social y el desarrollo (2009), se planteó que:

Ésta debería asumir el liderazgo social en materia de creación de conocimientos de alcance mundial para abordar retos mundiales, entre los que figuran la seguridad alimentaria, el cambio climático, la gestión del agua, el diálogo intercultural, las energías renovables y la salud pública.

De esto se puede inferir, que dadas las actuales circunstancias, las exigencias que se le presentan a la educación universitaria, desproporcionan sus ámbitos de gestión y los esquemas utilizados por los actores académicos para llevar a cabo su trabajo; de esta manera, el movimiento del mundo y de la cultura han modificado el sentido de la vida universitaria, multiplicando sus significados, o en otros casos, desvaneciéndolos mediante el impacto de los cambios; lo que además, ocasiona desplazamientos y genera rutas diferentes para realizar la inestable misión institucional.

Como Sancho, Creus y Padilla (2010:32) lo han expresado:

Hace unos años el profesorado universitario dedicaba su tiempo aparentemente tranquilo a enseñar un conocimiento relativamente estable y legitimado a unos estudiantes aparentemente predispuestos a aprender. Complementaba su trabajo, en algunos casos, con el desarrollo de alguna investigación y la publicación de algún artículo o monografía. En este momento, ha de dedicar una considerable cantidad de 
tiempo a enseñar un conocimiento emergente y cambiante a un alumnado diversificado y con necesidades muy diferentes.

Entre tanto, el control sobre el tiempo de trabajo docente y la distribución de asignaciones o tareas académicas, también ha pasado a formar parte de estilos de fiscalización administrativa, como es el caso del empleo de sistemas biométricos al estilo fabril, una versión refinada del taylorismo, traída de los confines de la producción material de finales del siglo XIX y ajustada a nuestra época para el control del trabajo intelectual del profesorado. Desde otro ángulo de análisis, estas prácticas también pueden indicar una forma de prolongar el sentido del tiempo que caracterizó al hombre medieval; siendo el sol el referente físico y el sonido de las campanas, su significado espiritual.

De acuerdo con Rama:

En el complejo mundo de la educación superior, el profesorado universitario también experimenta la sacudida de una nueva realidad dada por un nuevo ciclo de estudios posteriores a los tradicionales estudios de grado, ha nacido como resultado de la complejidad de los procesos tecnológicos y de la notable expansión de los conocimientos derivados de las inversiones económicas. Se constituye como un nuevo ciclo de organización de los saberes derivado de la enormidad de conocimientos y de su mayor nivel de complejidad, profundidad, integración y especialización disciplinaria e interdisciplinaria (2009:45).

Una de las consecuencias de este proceso de transformación en el conjunto de saberes y conocimientos, lo constituye el aparecimiento de nuevas exigencias para el ingreso y permanencia en la profesión docente del nivel de educación superior, entre las cuales se pueden mencionar, la aprobación de estudios de postgrado, la formación continua, la participación en redes académicas de investigación, la práctica de nuevos enfoques didácticos, las relaciones de colaboración entre profesores, la recreación curricular, entre otras.

En el análisis crítico del trabajo docente, Gómez, Escofet y Freixa (2015: 209) afirman que: Los profesores deben ser personas sensibles y cuidadosas ya que la enseñanza es una tarea humana compleja que no puede reducirse a una técnica. Los profesores no deben considerarse a sí mismos como una élite de expertos, sino que deben involucrarse de forma responsable con las comunidades en las que ejercen su labor.

La popularización del conocimiento científico, el acceso a sus beneficios por parte de sectores de la población que han sufrido de la exclusión e inequidad social, constituyen finalidades éticas cuya concreción puede ser alcanzada a través de la inserción de la 
actividad universitaria en la vida cotidiana, transfiriendo los aprendizajes a situaciones y contextos socio - naturales; así como utilizando la investigación científica en la averiguación y solución de los problemas que enfrenta la población urbana y rural. Se trata entonces, de rebasar el simple instrumentalismo mediante el que algunas veces se comprende al conocimiento, para llenar las funciones sustantivas de la universidad de contenidos y finalidades sociales.

La dimensión docente, que por largo tiempo se constituyó como el principal asidero del trabajo universitario, ha necesitado salir de sus límites conceptuales y procedimentales, para imbricarse con la investigación científica con el propósito de renovarse o de apropiarse de sus herramientas en el desarrollo del proceso pedagógico que facilita el aprendizaje de los educandos; pero demás, requiere de la determinación de su pertinencia social a través de sus imbricaciones con la vida comunitaria; donde existen problemas, experiencias para su validación y datos para la reconstrucción de la enseñanza.

Como Vergara, Gamboa y Montes lo señalan:

El ejercicio investigativo concede al profesor mayor profundidad de conocimientos, permite valorar el papel que desempeña la investigación en el desarrollo de su vida, lo ayuda a mejorar sus métodos y resolver problemas educacionales. La investigación abre un nuevo e interesante mundo en el cual se puede experimentar un continuo mejoramiento personal y profesional y esto es una garantía de calidad para la institución (2014: 163).

Desde tal perspectiva, se puede distinguir entre la investigación científica fundamental y aplicada, como campo de trabajo individual, grupal o de redes de investigadores; cuyos descubrimientos permiten la creación de modelos, teorías, conceptos, fórmulas, métodos u otros elementos epistemológicos del corpus científico y que sirven para la constitución de la identidad de la academia universitaria; y la investigación formativa, que al ser utilizada en los procesos docentes, permite según Rojas:

Plantear problemas, ensayar miradas diversas sobre las cuestiones duras y blandas de la ciencia e invitar a otros a la recapitulación del conocimiento disponible. Esta investigación requiere hacerse responsable del discurso académico, sin que esto esté vinculado necesariamente con una formación programada de investigadores. (2011:127).

El replanteamiento de la función docente también debe incorporar el uso del método de la crítica pedagógica, a fin de reformular el significado de los aprendizajes, aún de aquellos cuya comprensión haya gozado de alta reputación didáctica y psicopedagógica, ya 
que los problemas sociales y los derivados del cambio climático, el progreso de la ciencia y la tecnología, y las mutaciones constantes del mundo del trabajo, provocan la continua emergencia de necesidades, fenómenos, hallazgos, instrumentos, paradigmas y soluciones; donde la provisionalidad y la incertidumbre se erigen como criterios de validación de lo que se aprende; sobre todo, cuando el mismo requiere ser transferido a situaciones novedosas e inesperadas, en las que el interés intrínseco en el conocimiento se torna insuficiente para satisfacer las visiones anticipadas en que se funda la teleología del proceso didáctico en el contexto del mundo globalizado.

\section{TENDENCIAS}

Las múltiples manifestaciones del mundo globalizado, la revolución científica y tecnológica, la integración cultural y económica, el cambio climático, la caída de las antiguas certezas, el poder desafiante de las incertidumbres, la exuberantes fuentes de aprendizaje, el paso a la metáfora de la red para darle sentido a la vida institucional y social; constituyen, entre otros, los elementos emergentes que modelan el diseño y desarrollo de las funciones sustantivas de la universidad.

Es a partir de ellos, como se marcan las tendencias sobre las cuales las instituciones de educación superior, establecen sus inquisiciones, su encuentro con las oportunidades y los desafíos, así como con las razones para atender la necesidad apremiante de aprender más sobre sí mismas y su entorno, valorar la pertinencia de lo que se enseña e investiga y establecer vínculos coherentes con la realidad social y natural. Esto implica la revisión y reconstrucción continua de los contenidos curriculares, los cuales se convierten en objeto de cuestionamiento y en fuente mediadora de la innovación; pero además, en un referente para que la gestión pedagógica universitaria proceda a diseñar e implementar programas de formación permanente en el profesorado.

Como expresa Rama:

Actualmente se ha pasado a un escenario donde se impone la necesidad de un proceso de actualización de los currículos y al tiempo la permanente actualización de las personas ya formadas y cuyos saberes básicos o especializados requieren de la actualización de las competencias para mantener sus propios ámbitos de trabajo (2009:44).

Otros de los elementos emergentes en el contexto de la educación superior del siglo XXI, se derivan del carácter cambiante del mundo laboral, el cual presenta nuevas 
exigencias sobre las cualificaciones profesionales; en tal sentido, los programas de formación profesional desarrollados por las carreras universitarias, deben buscar que los resultados de aprendizaje o las capacidades profesionales alcanzadas, posibiliten la incorporación adecuada en la vida laboral o en la adaptación a ella, puesto que de manera acelerada, los espacios de trabajo se han convertido en territorios donde los nuevos y viejos trabajadores se sienten amenazados, o en el más extremo de los casos, se ven desplazados por nuevos trabajadores, máquinas o procesos científico - tecnológicos. Al respecto, Jeremy Rifkin (1997) advierte que “una gran mayoría de trabajadores se sienten completamente faltos de preparación para asumir la transición a la que deben enfrentarse. La eclosión de los avances tecnológicos y de las iniciativas de reestructuración económica parece habernos invadido sin previo aviso.”

Por otro lado, se da la expansión acelerada de los sistemas de educación superior, la incorporación y uso de recursos tecnológicos en la docencia y la investigación, la participación activa en programas derivados de la internacionalización de la educación, el surgimiento y consolidación de la educación terciaria para el desarrollo de la formación técnica en personas jóvenes y adultas, las nuevas formas de relación entre las instituciones universitarias y las empresas, la investigación científica aplicada al fortalecimiento de la seguridad alimentaria, la provisión de servicios de salud y agua de calidad para la población; así como, el establecimiento de relaciones de respeto y solidaridad con los demás seres vivos.

\section{DESAFÍOS}

Considerando que las tendencias que se manifiestan a nivel local, regional y mundial determinan las rutas de trabajo que las universidades deben plantearse para desarrollar las funciones de docencia, investigación y vinculación con la sociedad, es atinente que la educación superior atienda la realización de indagaciones permanentes sobre las condiciones y exigencias que presenta el mundo laboral, la constitución de redes académicas para el mejoramiento de la docencia y el desarrollo de la investigación científica, valorando en cada caso, las diferencias particulares y la conveniencia de homogenizar procesos y resultados; la formación permanente del profesorado, que incluya el dominio científico de la disciplina y el uso de enfoques didácticos que posibiliten la transferencia de lo aprendido a otros contextos y situaciones; el desarrollo de actividades 
curriculares y de vinculación social en las que se visibilice la interdependencia y respeto entre los seres vivos, así como el fomento de valores democráticos, de transparencia y vocación de servicio.

Otro de los desafíos concurrentes en el desarrollo de la educación superior, lo constituye la necesidad de implementar programas de realfabetización digital del profesorado; entre otras razones, se puede decir que su necesidad se justifica en la idea expresada por Tomas et al (2010:20) cuando dice:

En la sociedad de la información y el conocimiento las universidades están destinadas a crear, criticar y difundir conocimientos haciendo uso de los medios y recursos tecnológicos que complementarán los métodos y recursos pedagógicos para poder llegar a la diversidad de estudiantes.

\section{PROPUESTA}

Las funciones sustantivas de las instituciones de educación superior deben establecerse sobre la base de un modelo holista que posibilite:

\section{a.- El fomento del trabajo interdisciplinario en las distintas dimensiones del desarrollo} y transformación de la cultura académica universitaria

La forma en que se organiza el conocimiento en la composición de las prescripciones curriculares, es uno de los elementos centrales para la planificación y desarrollo del trabajo académico que se lleva a cabo en los distintos espacios de la actividad universitaria; en tal sentido, sirve como referente en las continuas lecturas que los docentes y autoridades de departamentos y facultades realizan al interpretar el sentido de la enseñanza.

Usualmente, esta organización ha estado basada en las estructuras particulares de las disciplinas, las cuales se han comprendido como "categorías organizacionales en el seno del conocimiento científico, de la que parte la división y especialización del trabajo” (Arango: 1999). En la práctica, esta definición se ha llevado al exceso y ha provocado la generación de experiencias en las que la relación con los objetos de estudio y su contextualización, se caracterizan por mantenerse en una situación parcelada y atomizada; lo cual, permite explicar los casos en que se da la ausencia del trabajo interdisciplinario en las instituciones de educación superior y a delatar la escasa formación epistemológica de algunos actores académicos para comprender el carácter esencialmente unitario del saber humano. 
El estilo de trabajo balcanizado que se desarrolla en varios escenarios que conforman la educación superior, tiene sus raíces en la influencia de corrientes de pensamiento tales como el positivismo de Comte y sus seguidores; así como en prácticas pertenecientes a la actividad empresarial, desde las que se erigen modelos como el taylorismo y el fordismo, los cuales han impregnado de su instrumentación a la cultura universitaria centroamericana y de otras regiones del mundo.

Comte (1984: 57) indicó que:

Hay que distinguir, en relación a todos los órdenes de fenómenos, dos clases de ciencias naturales: unas abstractas, generales, que tienen por objeto el descubrimiento de las leyes que rigen las diversas clases de fenómenos, considerando todos los casos que se puedan imaginar; las otras, concretas, particulares, descriptivas.... que consisten en la aplicación de estas leyes.

De esta manera, logró establecer una clasificación de la ciencia en las que incluye a la biología, matemática, física, astronomía y física social (nombre con el que originalmente se le llamó a la sociología), delimitando las fronteras de cada campo de estudio, pero a la vez, incorporando una visión jerárquica en la comprensión de las mismas; lo que sirve incluso, para explicar la vigencia que actualmente tienen algunos criterios utilizados en la temporalización de los espacios académicos y en la definición de los créditos o unidades valorativas prescritas en el pensum académico de las carreras universitarias.

Asimismo, sostuvo que los conocimientos sobre la naturaleza y los procedimientos que de ellos se deducen para modificarla en nuestro beneficio, deben concebirse como dos sistemas esencialmente distintos en sí mismos y abordados por separado. En tal sentido, afirmó que "todos los trabajos humanos son o de especulación o de acción. Así pues, la división más general de nuestros conocimientos reales consiste en distinguirlos en teóricos y en prácticos” (1984: 53).

La generalización argumentativa establecida por Comte ha servido como base para alimentar la escisión entre teoría - práctica en la vida universitaria, lo cual ha generado algunas implicaciones tanto en las maneras de lograr los aprendizajes promovidos desde las asignaturas, módulos y seminarios que ocupan el mayor tiempo y espacio en la actividad docente, como en los enfoques utilizados para diseñar y desarrollar la investigación científica y las formas de vinculación con la sociedad; provocando que las funciones sustantivas de la universidad aniden en compartimientos estancos y desprovistas 
de contexto. Esto permite que en el pensamiento académico universitario, se puedan identificar esquemas unilaterales en las formas de comprender la relación teoría - práctica; así como el surgimiento de conductas académicas alienadas, empotradas en la autosuficiencia de una de las partes de dicha vinculación.

Hay que reconocer que Comte advirtió sobre la necesidad de "evitar los perniciosos efectos de la excesiva especialización sobre el porvenir intelectual” (1994:37), no obstante, su propuesta de perfeccionar el trabajo intelectual en sí mismo y la constitución del estudio de las generalidades científicas en una gran especialidad nueva, no le alcanzó para contrarrestar la diseminación de abundantes concepciones arborescentes en el mundo científico.

Por su parte, del modelo fordista - taylorista se han adoptado pautas para la organización del trabajo en las instituciones de educación superior, la cual se caracteriza por la fragmentación en el desarrollo de las tareas, el control de los tiempos y la separación entre concepción y ejecución. Estas expresiones pueden ser identificadas con algún grado de facilidad, en la estructura organizativa de las facultades y departamentos, en los diseños curriculares y en las concepciones científicas predominantes en los docentes.

Es oportuno añadir, que la fuerza de estas tradiciones también constituye uno de los principales obstáculos que se dan en algunas universidades, para alcanzar la concreción de los planes de estudio con enfoque basado en competencias; puesto que los profesores interpretan el proceso educativo desde la particularidad de su asignatura, sin alcanzar a comprender el sentido de la integración de los contenidos conceptuales, procedimentales y actitudinales, ni a establecer relaciones de interdependencia con otros saberes y áreas de conocimiento; cuestión que resulta crucial para el desarrollo de las competencias en el plano del desarrollo curricular y para la nueva configuración de las funciones sustantivas de la universidad en el plano de la cultura institucional.

Eisner (1998: 275) sostiene que:

Una disciplina es un instrumento, un producto de la imaginación diseñado para servir a alguna función útil...Los niveles de abstracción y el tema de la disciplina son arbitrarios. Podemos dividir el mundo de cualquier manera que le dé sentido. Menciono esto porque algunas personas creen que las disciplinas son entidades naturales en lugar de instrumentos culturales. 
El carácter cultural que Eisner le atribuye a las disciplinas, ayuda a entender que la constitución de su cuerpo conceptual y metodológico está sometida a los cambios que se dan en la historia de la ciencia y del mundo, que sus objetos de estudio y fronteras pueden sufrir modificaciones; en consecuencia, la acostumbrada invocación a lo absoluto, utilizada para alcanzar los niveles de seguridad en la explicación y verdad científica, es cada vez más invadida por la relatividad que genera el cambio; y, entre tanto, en la sociedad de nuestro tiempo también se da el advenimiento de elementos emergentes que desafían a la autoridad científica.

Algo similar, ocurre con los contenidos desde lo que estructuran las funciones sustantivas de la universidad y con las formas de actuación profesional de los miembros de la comunidad académica que la conforma; por esto, resulta muy atinada la cita que Villagra (2011:39) hace sobre algunos de los planteamientos de Brecher, en los que expresa que:

La declarada y declamada interdisciplinariedad lejos de su unívoca e indiscutible aceptación, constituye una de las batallas entre tribus académicas más difíciles de superar en el ámbito universitario. El trabajo entre distintos profesionales suele transformarse simbólicamente en un campo de perpetuo enfrentamiento, muy a distancia de los aspirados espacios de debate y construcción cooperativa.

Las ideas y prácticas separatistas que por largo tiempo han acaparado la forma de hacer y rehacer la vida académica de las universidades, no alcanzan a comprender que el encierro en el propio ámbito de especialización disciplinar se llega a convertir en un marco de simplificaciones o en un terreno desgastado que requiere ser enriquecido a través de las transferencias reciprocas que tienen lugar en el trabajo interdisciplinario; entre tanto, tampoco se ahonda en la reflexión, ni se da paso a las intersecciones necesarias para que la docencia, la investigación científica y la vinculación con la sociedad sean laboratorios naturales para el propio perfeccionamiento profesional de los docentes universitarios.

Al respecto, Souza (2008) expresa que:

La postura y el movimiento en la dimensión interdisciplinaria permite tomar la perspectiva del proyecto, al proyectar la docencia universitaria se encuentra la posibilidad de construir nuevos sentidos para la formación de profesores que tienen en la enseñanza superior su terreno de trabajo y producción científica. En estos nuevos sentidos se destaca la búsqueda por configurar espacios de aprendizaje que estén relacionados íntimamente con las necesidades y demandas sociales, respondiendo a los desafíos contemporáneos de la ética, de la crítica y de la ciudadanía. 


\section{b.- La bioética como espacio de trabajo transdisciplinario y su significado en la}

integración de las funciones sustantivas de la universidad

La identificación y comprensión de la bioética como uno de los espacios para el trabajo científico transdisciplinario, es un intento por aprovechar las infinitas posibilidades que ofrece para integrar valores, conocimientos, paradigmas y métodos provenientes de distintas fuentes de la cultura, entre las que se pueden mencionar: las disciplinas que conforman el corpus académico de las carreras, las disciplinas que existen fuera de la vida universitaria y las disciplinas que están en proceso de construcción en el mundo académico, empresarial y cotidiano; las que, por su condición, aún no son explícitas en los contextos de argumentación y práctica correspondiente; pero, más allá de su investidura y situacionalidad, al formar parte del análisis y recreación transdisciplinar, las disciplinas viven su integración en el rebasamiento de sus propios fronteras, lo cual constituye una de las condiciones ontológicas para transformarse en algo distinto.

En este orden de ideas, se puede decir que este es uno de los puntos de partida para considerar que en la dinámica que de esta manera se despliega, se pueden encontrar rutas de pensamiento y actuación que se tornan necesarias para la reconfiguración continua de las funciones sustantivas de la universidad; para lo cual, se requiere que las instituciones de educación superior, además de mirar hacia sí mismas (normativas, programas y procesos) deben mirar hacia el mundo, para favorecer el encuentro con lo cotidiano, en el que prolifera lo diverso y múltiple, y sobre lo cual, la academia universitaria debe efectuar constantes actos recursivos de autoconcepción, para pensarse y sentirse legítima.

Si bien, la cultura universitaria está dotada de contenidos científicos, tecnológicos, filosóficos, literarios y artísticos; hay que reconocer que en el mundo hay otros contenidos necesarios para la vida de los seres que habitan la tierra, por básicos y muy de sentido común que parezcan, también son elementos en los que se fundan relaciones de interdependencia y fructificación. En este sentido, hay que admitir con Kosik (1967: 25) que:

La actitud que el hombre adopta primaria e inmediatamente hacia la realidad no es la de un sujeto cognoscente, o la de una mente pensante que enfoca la realidad de modo especulativo, sino la de un ser que actúa objetiva y prácticamente, la de un individuo histórico que despliega su actividad práctica con respecto a la naturaleza y a los hombres. 
Y es quizá, en este marco de representaciones y prácticas donde empiezan los procesos históricos de la ciencia y la creación inacabada de conocimientos que a través de la sistematización, alcanzan a estructurarse como dimensiones de la actividad disciplinaria. Los distintos significados que desde su surgimiento se le han atribuido a la bioética, pueden generar posiciones dispares o divergentes respecto a la fuerza heurística que se manifiesta en los procesos de integración del conocimiento y la creación de nuevas identidades epistemológicas.

$\mathrm{Y}$ es, precisamente, en la afluencia de definiciones sobre su quehacer fundamental, donde se pueden identificar distintas dimensiones semánticas e instrumentales, mediante las que se le concibe como rama de la ética encargada del estudio de cuestiones médicas y sanitarias; asimismo, desde su sentido teleológico, se declara que su finalidad consiste en establecer criterios, normas y principios que guíen el obrar del hombre respecto a la vida; como interpretación de las tendencias del siglo XXI, se le define como la conciencia crítica de la civilización tecnológica; pero, además, desde visiones más amplias con implicaciones holistas, se sostiene que es el estudio de todos los problemas de la vida en general, el medio ambiente y el debido trato a los animales; así mismo, se expresa, que la bioética abarca las cuestiones éticas acerca de la vida que surgen en las relaciones entre biología, nutrición, medicina, química, política, derecho, filosofía, sociología, antropología, teología y otros campos del conocimiento; a la bioética también se le concibe como el uso creativo del diálogo inter y transdisciplinar entre ciencias de la vida y valores humanos para formular, articular y, en la medida de lo posible, resolver algunos de los problemas planteados por la investigación y la intervención sobre la vida, el medio ambiente y el planeta Tierra.

Como puede apreciarse, en este conjunto de definiciones concurren diversas concepciones sobre el significado y lugar que ocupa la bioética en el cuerpo unitario que constituye el pensamiento humano, y en las que se marcan sesgos monodisciplinarios, interdisciplinarios y transdisciplinarios; cuya validez debe aceptarse como parte necesaria de la composición lógica y ética del debate universitario. No obstante, es su estructuración como campo transdisciplinario, el objeto pensado desde esta propuesta, pues como expresa Edgar Morín:

La transdisciplinariedad representa la aspiración a un conocimiento lo más completo posible, que sea capaz de dialogar con la diversidad de los saberes humanos. Por eso 
el diálogo de saberes y la complejidad son inherentes a la actitud transdisciplinaria, que se plantea el mundo como pregunta y como aspiración.

A través de las reflexiones teóricas expresadas, se pretende fundamentar la manera en que la multiplicidad de contextos, perspectivas, metodologías, relaciones, hallazgos y tareas en que se establece la bioética; pueden contribuir en la integración de las funciones sustantivas de las instituciones de educación superior, en las cuales, las nuevas configuraciones se podrán construir ejerciendo un control cognitivo sobre los elementos emergentes en el entorno global y local, e instaurando relaciones con la realidad social y natural, comprendida ésta, como fuente generadora de las grandes interrogantes de la investigación científica y de la renovación de los aprendizajes, sin perder la ruta de humanizar el mundo y sin atropellar los derechos de los demás seres vivos.

Desde otra perspectiva y conviniendo con las palabras de Bellver (2014:3):

Actualmente existe una diferencia de casi 25 años en la esperanza de vida entre los países más desarrollados y los más pobres del mundo. Para reducir ese abismo bastaría con que se garantizara a los ciudadanos de esos países la satisfacción de algunas de sus necesidades básicas, como el saneamiento, el acceso al agua potable, la seguridad alimentaria, la asistencia sanitaria básica o la protección frente a los desastres naturales y las catástrofes.

Pero, en el mismo cuerpo del texto, este autor continúa con su inquisición, advirtiendo que “el desafío es lograr que el incremento de la esperanza de vida traiga consigo un incremento de la esperanza de vida saludable y no la mera prolongación de la decrepitud de las personas”.

Los caminos para la integración de las funciones sustantivas de la universidad son variados e inacabados, por tal razón, se requiere asumir actitudes de respeto y tolerancia entre los miembros de la comunidad universitaria; así como, en las relaciones de ésta con la sociedad, para dar paso a la indagación constructiva y a la posibilidad de ejercer una autocrítica consciente sobre el propio trabajo; sea este individual, de equipos o de redes.

El progreso de la ciencia y la tecnología, así como los nuevos problemas y discusiones que atienden la ética y la epistemología, o en su conjunto, los distintos campos del conocimiento existentes dentro y fuera del ámbito universitario; son una especie de caños que sirven para que la creatividad y práctica académica genere nuevos espacios de trabajo en los que se transita de la anormalidad o excepcionalidad a la normalidad de la explicaciones y soluciones encontrados en los procesos de descubrimiento y renovación; por esto, no faltarán ocasiones en que en las comunidades académicas se requiera 
fundamentar “esa otra cosa que no existía”, que genera asombro o interés defensivo por conservar lo ya validado.

Esta reflexión sobre lo novedoso, lleva para el caso, a la interpretación de Adela Cortina, en la plantea que:

La ética de la neurociencia intenta desarrollar un marco ético para regular la conducta en la investigación neurocientífica y en la aplicación del conocimiento neurocientífico a los seres humanos. En este sentido, podría considerarse simplemente como una rama especializada de la bioética (Marcos, 2015:406).

Es importante resaltar, que la opción de integración interdisciplinaria propuesta, puede ayudar en la convocatoria para iniciar acercamientos entre iniciados, expertos y ciudadanos que permitan la construcción científica y social de la vida académica y por supuesto, el mejoramiento cualitativo de la enseñanza.

\section{c.- El fortalecimiento de la relación heurística entre docencia, investigación y vinculación social}

La comprensión de las funciones sustantivas de la universidad como espacios de trabajo en los que se establecen relaciones de interdependencia, hace posible la aplicación o práctica de estrategias heurísticas para la generación de descubrimientos a través de los que se enriquecen los aprendizajes, se fomentan la producción de conocimientos y se impulsa la solución de problemas a nivel institucional y comunitario.

En los dos apartados anteriores, se ha resaltado la importancia que tienen las formas de integración del conocimiento por las vías inter y transdisciplinaria, con el propósito interpretar el carácter que presentan los elementos emergentes que condicionan la actividad universitaria en el presente siglo, para luego, buscar la estructuración de alternativas para la nueva configuración de su identidad y esencia transformadora.

Por otra parte, es importante reconocer, que el acto mediante el cual la comunidad universitaria establece vínculos con la sociedad; implica, en cierta medida, una manera de renunciar a sí misma, para lograr que se dé el efecto de impregnación recíproca, la flexibilización en los procesos de vinculación y la recreación del sistema de ideas en el que se fundamenta el significado de su lugar y su no lugar en los distintos contextos de pensamiento e intervención social en que se da la objetivación de sus funciones sustantivas.

En consecuencia, precisa que a la linealidad con que se concibe y diseña la investigación científica y las relaciones con la sociedad, se agregue una visión permeable a 
las divergencias y en la cual, haya lugar para que la creatividad se manifieste entre el conjunto de los saberes elaborados por el pensamiento humano, sean estos sistemáticos o no; es así como, la aplicación de estrategias heurísticas pueden llegar a favorecer la activación de las continuas salidas, retornos y recomposiciones en los dominios teóricos, metodológicos y prácticos de la docencia, la investigación científica y la vinculación que las universidades establecen con la sociedad.

Los caminos para inquirir, sistematizar y dotar de sentido a tal propuesta pueden asumirse desde distintas perspectivas científicas y filosóficas; una de las que se puede mencionar a manera de referencia, y sin tratar de impulsar forma de adhesión alguna, más que aquella que surja de la crítica y de la libertad para pensar; es la concepción heurística desarrollada por Imri Lakatos (1989), en la cual, la concibe como medio para el descubrimiento y la innovación en los programas de investigación científica.

De igual forma, distingue entre la heurística negativa y la heurística positiva; en la primera, destaca que las tareas más convencionales que se realizan en el seno de la comunidad científica que abrazan los programas de investigación científica (PIC), corresponderían al afán de los investigadores en proteger la esencia del núcleo del programa de investigación; mientras que la heurística positiva, consiste en un conjunto parcialmente articulado de sugerencias o indicaciones sobre cómo cambiar y desarrollar las ‘variantes refutables’ del programa de investigación; cómo modificar y refinar el cinturón protector refutable.

Más allá de la discusión filosófica que a partir de estos planteamientos se pueda desarrollar, la idea de comentarlos, responde al propósito que tiene esta ponencia, en el sentido de provocar reflexiones o llamar la atención sobre algunos aspectos de la vida académica universitaria que pueden servir como puntos de partida para transformar la visión tradicional de la docencia y la investigación científica, así como la conducta extensionista que las instituciones han manifestado durante largo tiempo hacia la comunidad; para situar en su lugar, las estrategias que permitan la transformación constructiva de las funciones sustantivas de las instituciones de educación superior, como apunta Bolívar y Caballero (2015), “el trabajo debe hacerse público y no permanecer en lo privado; hablaríamos pues de una enseñanza visible que abra las puertas del aula al exterior”. 


\section{REFERENCIAS BIBLIOGRÁFICAS}

Arango, A. (1999). El taller de interdisciplinariedad como práctica educativa para el trabajo comunitario para el área de la salud. Memoria, Tomo I, (X Congreso Internacional de Tecnología y Educación a Distancia). San José: EUNED.

Bellver, V. (2014). Ética y políticas ante la investigación para extender la vida humana. Cuadernos de bioética. Valencia: Departamento de Filosofía del Derecho, Moral y Política, Universidad de Valencia.

Bolívar, A. y Caballero, K. (2015). El profesorado universitario como docente: hacia una identidad profesional que integre la docencia y la investigación. REDU. Revista de docencia universitaria. Vol. 13 (1). España: Universidad de Granada.

Comte, A. (1984). Curso de filosofía positiva. Barcelona: Orbis.

Eisner, E. (1998). El ojo ilustrado. Indagación científica y mejora de la práctica educativa. Barcelona: Paidós.

Gimeno Sacristán, J. (2001). Educar y convivir en la cultura global. Madrid: Morata.

Kosik, K. (1967). Dialéctica de lo concreto. México: Grijalbo.

Lakatos, I. (1989). La metodología de los programas de investigación científica. Madrid: Alianza Editorial.

Marcos, A. (2015). Neuroética y vulnerabilidad humana en perspectiva filosófica. Cuadernos de bioética. Valladolid: Universidad de Valladolid.

Morín, E. (s/f). ¿Qué es la transdisciplinariedad? Recuperado de: http://www.edgarmorin.org/que-es-transdisciplinariedad.html

Rama, C. (2009). La universidad latinoamericana en la encrucijada de sus tendencias. Tegucigalpa: UNAH.

Rifkin, J. (1997). El fin del trabajo. Nuevas tecnologías contra puestos de trabajo: el nacimiento de una nueva era. Barcelona: Paidós.

Rojas, H. (2011). Docencia y formación científica universitaria. Revista Magis, Vol.4, No.7, Bogotá. Recuperado http://magisinvestigacioneducacion.javeriana.edu.co/

Sancho, J., Creus, A. y Padilla, P. (2010). Docencia, investigación y gestión en la universidad: una profesión, tres mundos. Praxis. Revista de investigación científica. Vol. XIV, No. 14. Editorial Universidad Real de la Pampa. 
Souza, H. (2008). Interdisciplinariedad, docencia universitaria y formación. Recuperado de http://bvs.sld.cu/revistas/ems/vol22_4_08/ems07408.htm

Tomas, M., Borrell, N., Castro, D., Feixas, M., Bernabeu, d. y Fuentes, M. (2010). La cultura innovadora de las universidades: estudio de casos. Ediciones Octaedro.

UNESCO. (2009). Conferencia Mundial sobre Educación Superior en el siglo XXI: Visión y acción.

Vergara, D., Gamboa, A. y Montes, A. (2014). Calidad de la educación superior: Un análisis de sus principales determinantes. Saber, ciencia y libertad. Vol.8, No.2.

Villagra, M. A. (2011). La didáctica universitaria o los avatares en la búsqueda de un lugar. Revista de educación. Año 2, No.3. Recuperado de http://fh.mdp.edu.ar/revistas/index.php/r_educ/article/viewFile/44/88 